\section{General practitioner attitudes and confidence to deprescribing for elderly patients}

Pier Riccardo Rossi, ${ }^{1-3}$ Sarah E. Hegarty ${ }^{4}$ Vittorio Maio, ${ }^{5}$ Marco Lombardi, ${ }^{6}$ Andrea Pizzini, ${ }^{1-3}$ Aldo Mozzone, ${ }^{1-3}$ Marzio Uberti, ${ }^{1-3}$ Simonetta Miozzo ${ }^{1,2,7}$

${ }^{1}$ Scuola Piemontese di Medicina Generale, Torino, Italy; ${ }^{2}$ Società Italiana di Medicina Generale e delle Cure Primarie, Torino, Italy; ${ }^{3}$ General Practitioner, Torino Local Health Authority, Torino, Italy; ${ }^{4}$ Division of Biostatistics, Department of Pharmacology and Experimental Therapeutics, Thomas Jefferson University, Philadelphia, PA, USA; ${ }^{5}$ College of Population Health, Thomas Jefferson University, Philadelphia, PA, USA; 'Parma Local Health Authority, Parma, Italy; ${ }^{7}$ General Practitioner, Pinerolo-Collegno Local Health Authority, Pinerolo-Collegno (TO), Italy

\section{Abstract}

Deprescribing is a patient-centered process of medication withdrawal intended to achieve improved health outcomes through discontinuation of one or more medications that are either potentially harmful or no longer required.

The objective of this study was to assess the perceptions of primary care physicians on deprescribing and potential barriers to deprescribing in the Local Health Authority (LHA) of Turin, Piedmont, Italy. Secondary objective was to evaluate educational needs of primary care physician.

Cross sectional survey of primary care physicians working in the LHA of Turin, Piedmont, Italy.

439 GPs $(71.3 \%$ of the total number of primary care physicians) attended an educational session related to deprescribing and were asked to anonymously answer a paper survey. Participants were asked to complete a previously published questionnaire about deprescribing and potential factors affecting the deprescribing process.

A correlation coefficient was calculated to assess the association between physicians' confidence in deprescribing and attitudes or barriers associated with deprescribing.

Many GPs (71\%) reported general confidence in their ability to deprescribe. Most respondents (83\%) reported they were comfortable deprescribing preventive medications, however almost half expressed doubts regarding deprescribing when medication was initially prescribed by a colleague $(45 \%)$ or when patient and/or caregiver supported the opportunity to continue the assumption (49\%). Around a third of doctors maintain that the absence of strong evidence supporting deprescribing prevents them from considering it (38\%), that they do not have the necessary time to effectively go through the process of deprescribing (29\%), and that fear of possible effects due on withdrawal prevents them from deprescribing (31\%). There was no strong correlation between physicians' confidence and attitudes or barriers associated with deprescribing.

The present study confirms that general practitioners sense the importance of deprescribing and feel prepared to face it managing communication with patients and caregivers, but find barriers when enacting the practice in a real-life context.

\section{How this fits in}

Data about physicians' confidence and attitudes toward deprescribing are limited. This study found that general practitioners recognize the importance of deprescribing and feel comfortable to undertake the deprescribing process with patients and caregivers. However, several barriers when enacting the practice in a real-life context were identified. Study results can be used to plan educational and training activities for primary care physicians and other health care professionals involved in the medication prescription process, as well as to design strategies for improving patients' understanding of appropriate use of medications. In addition, these results can provide useful elements for political decision-makers and for those who are delegated to organize healthcare services for the elderly.

\section{Introduction}

Polypharmacy is an ever-growing healthcare issue, mainly common in elderly patients. While it can sometimes be necessary and motivated by multimorbidity, ${ }^{1}$ polypharmacy can still represent an issue for at least two main reasons: the risk of pharmacological interactions and adverse reactions (ADR) on the one hand and a decrease in compliance (adherence to the therapy) on the other hand. Consequences can be excessive hospitalization because of
Correspondence: Pier Riccardo Rossi, Scuola Piemontese di Medicina Generale; Società Italiana di Medicina Generale e delle Cure Primarie; General Practitioner, Torino Local Health Authority, via Emilio Ghione 40, 10156 Torino, Italy.

E-mail: ric.rossi@hotmail.it

Key words: Deprescribing; general practitioner; attitude; primary care; elderly.

Acknowledgments: we thank for their contribution the following tutors of the Scuola Piemontese di Medicina Generale: Caposieno M, Araldi M, Boella G, Fassone R, Piano P, Torta F, Morato P, Di Gravina G, Rumore A, Vitali S, Mandas R, Bianchi S, Raiteri G.

Contributions: the authors contributed equally.

Conflict of interest: the authors declare no potential conflict of interest.

Received for publication: 2 December 2019. Revision received: 19 January 2020.

Accepted for publication: 24 Janauary 2020.

This work is licensed under a Creative Commons Attribution-NonCommercial 4.0 International License (CC BY-NC 4.0).

${ }^{\circ}$ Copyright: the Author(s), 2020

Licensee PAGEPress, Italy

Geriatric Care 2020; 6:8703

doi:10.4081/gc.2020.8703

detrimental pharmacological interactions, falls, decreased cognitive function, ${ }^{2,3}$ and the decrease in potential pharmacological benefits.

Guidelines motivating drug prescription derive from case studies on single pathologies and in most cases on selected populations: this approach immediately excludes polymorbid and/or elderly patients. ${ }^{4-6}$ It can therefore be said that polypharmacy on elderly patients often represent isolated experiments. ${ }^{7}$ The challenge is to establish, based on each individual patient, whether it is possible to introduce a new medication or to deprescribe one based on the current health situation while also respecting the patient's and caregiver's preferences.

Polypharmacy must, therefore, be monitored and constantly adapted to the patient's needs over time. All healthcare practitioners should consider the positive and negative potential of polypharmacy, but the best-suited figures to take care of the problem are General Practitioners (GPs) who operate in the context of primary care and are ultimately in charge of caring for the person and have knowledge of their history and quality of life. 
Deprescribing has recently been defined as follows: a patient-centered process of medication withdrawal intended to achieve improved health outcomes through discontinuation of one or more medications that are either potentially harmful or no longer required. ${ }^{8}$ Such a process can very well be referred to the concept of Quaternary Prevention (P4). ${ }^{9} \mathrm{P} 4$ is defined as: Actions taken to identify a patient or a population at risk of over-medicalization, to protect him/her from invasive medical procedures, and to offer them ethically and medically acceptable treatment procedures. ${ }^{10} \mathrm{P} 4$ is a critical look at medical activities with an emphasis on the need not to harm. ${ }^{11}$ and is consider by WONCA a task for GPs. ${ }^{9}$ Some studies evaluated the effectiveness of deprescribing, finding improved quality of life and no association to significant risks or withdrawal symptoms. ${ }^{12,13}$ Of the potential benefits, there are still many obstacles that make deprescribing difficult for physicians. Some studies highlight how the lack of time, difficulty communicating with caregivers, patients and other healthcare practitioners, fragmental medical care, patients' and physicians' conservatism/inertia and the lack of guidelines for suspension criteria can hinder the process. ${ }^{14-17}$ Evidence suggests that using a patient-centered approach and including patient's perspective into the decision making process are key elements for deprescribing in the elderly population..$^{18}$

The present study aims to determine whether the general practitioner's perception and recognition of obstacles could potentially hinder the use of the deprescribing process. A secondary aim is to detect eventual educational needs of general practitioners or organisational deficiencies within the field of primary care giving.

\section{Materials and methods}

The study is based on a cross sectional survey. The population studied is made up of GPs working in the Local Health Authority (LHA) of Turin, Piemonte, Italy. The population is made up of 616 doctors entrusted in the care of a population of about 900,000 people. We employed a published questionnaire developed and used for a similar research conducted in the LHA of Parma, Italy. ${ }^{19}$ We received the original Italian version of the nine-item questionnaire from the authors. In essence, the questionnaire was designed to evaluate attitudes and detect levels of confidence in doctors regarding deprescribing for elderly patients. The nine statements in the questionnaire explore deprescribing issues such as suspension of a drug for either preventive or therapeutic use, the ability to motivate patients towards deprescribing, and the barriers to deprescribing. Doctors were asked to indicate the degree to which they agreed with the nine statements using a Likert-type scale ranging from 1 (highly disagree) to 7 (strongly agree).

During the year 2018 a group of tutors instructed by the Scuola Piemontese di Medicina Generale (School of General medicine in Piedmont) developed and presented an educational program on deprescribing sponsored by the Turin LHA. The seminars, held over the course of two evenings, were mandatory and took place within the context of monthly team meetings from April 2018 to November 2018. The questionnaire was voluntary and anonymous and was presented at the beginning of the second evening (October-November 2018). All answers were collected on paper and then keyed into an excel spreadsheet.

Descriptive statistics were calculated to summarize responses to the nine items. Pearson's correlation coefficients were calculated to determine the relationship between the first question (Q1) on the doctor's faith in deprescribing and all other questions regarding attitudes or barriers associated with deprescribing. To simplify interpretation, the original 7-point scales was dichotomized; responses of 5, 6 and 7 strongly agree were grouped in one category declaration of agreement, while items 1 strongly disagree, 2, 3 and 4 were grouped in a declaration of disagreement category. Associations between demographic information about GPs (age, sex, specialization) and agreement or disagreement on each question in the survey were evaluated using logistic regression. A value of $\mathrm{P}<0.05$ has been considered statistically relevant in all analyses. All analyses were conducting using SAS 9.4 (SAS Institute Inc., Cary, NC, USA).

\section{Results}

A total of 439 GPs completed the survey (71.3\% of the total number of primary care physicians in Turin). The average age was 59.1 years (SD: 6.1). A little over half of GPs were women (51.4\%) (Table 1). 32\% of GPs reported a specialization. Complete answers to the survey are reported in Table 2 . The statement with the highest average agreement score was: When the life expectancy of my elderly patients no longer justifies potential benefits, I am in favor of deprescribing preventive medications. Nearly $70 \%$ of GPs feel prepared to tackle deprescribing, however almost half expressed doubts regarding deprescribing when medication was initially prescribed by a colleague $(45 \%)$ or when patient and/or caregiver supported the opportunity to continued use of said drugs (49\%). Around a third of doctors maintain that the absence of strong evidence supporting deprescribing prevents them from considering it (38\%), that they do not have the necessary time to effectively go through the process of deprescribing (29\%), and the fear of possible effects brought on from the interruption of said drugs could possibly prevent them from deprescribing (31\%). (Table 3).

Correlation analyses between the first statement and other statements revealed a weak correlation between perceived level of expertise and the ability to deprescribe medication initially prescribed by another colleague (fifth statement, rho $=0.33$ ) and the ability to motivate the patient to begin the process of deprescribing ( ninth statement, rho $=0.33)$ (Table 4.) Overall, there was no statistically significant association between sex of the participants and the probability that they will agree to any of these statements. However, older doctors (over 60) show a greater willingness to agree with the following statements: In elderly patients, lack of robust evidence in favor of continuation or cessation of preventive medications prevents me from deprescribing $(\mathrm{P}=0.003)$, and Although in certain situations I may consider appropriate deprescribing medications in my elderly patients, I do not consider it for fear of adverse drug withdrawal effects $(\mathrm{P}=0.046)$. Specialised doctors show less willingness, compared to other colleagues, to agree with the statement: I do not have the necessary time to spend with my elderly patients and/or caregivers to effectively undertake the process of deprescribing medications even though I consider it important $(\mathrm{P}=0.008)$ (data not shown).

\section{Discussion \\ Summary}

The results of the present study confirm that there are inconsistencies between actual understanding and perceived knowledge from prescribers regarding the process of deprescribing and the ability to put the process into action. ${ }^{19}$ In fact, even though most doctors declare to understand and agree with the concept of deprescribing, the responses to statements regarding their approach to it and its difficulties demonstrate the presence of a certain hesitation or difficulty in tackling and bringing into action the completion of deprescribing while also 
facing the difficulties presented by the context of daily practice.

\section{Strengths and limitations}

This study expands our knowledge on physicians' perception on deprescribing and related barriers. The high survey response rate makes the study results robust. However, there are some limitations worth noting. The survey was administered to primary care physicians in a specific LHA within Italy, and therefore the results cannot be generalized to the overall population of primary care physicians in Italy or elsewhere. Data were self-reported and therefore subject to bias. Despite a high response rate, we do not have responses of other GPs in Turin LHA who chose not to participate and, thus, cannot evaluate their beliefs for consistency with the study sample. Those GPs who decided to complete the questionnaire may have been more interested in the subject of deprescribing than those who decided not to participate. Lastly, limitations of the survey tools exist as they were previously reported by its authors: ${ }^{19}$ the small number of items may not be able to explore a complex and multifaceted problem such as physician's attitude to deprescribing. Additionally, wording for some questions may have become complex and subject to multiple interpretations.

\section{Comparison with existing literature}

Previous research has demonstrated the effectiveness of the process in the reduction of outcomes such as mortality, hospitalization, falls, cognitive impairment; ${ }^{16-20}$ nonetheless, in practical terms, deprescribing is limited by the fear of adverse effects caused by discontinuation, beliefs of the patient or caregiver, or fear of clashing with other doctor's prescription.

Most doctors expressed agreement with deprescribing preventive medication; however, fewer agreed with deprescribing guideline-recommended therapeutic medications in patients with low life expectancy. When comparing the two

Table 1. Characteristics of respondents $(\mathrm{N}=439)$.

\begin{tabular}{lcc} 
Age, mean (SD) & & $(6.1)$ \\
Age, N (\%) & 59.1 & $(4.7)$ \\
$<50$ & 20 & $(41.6)$ \\
$50-59$ & 179 & $(53.7)$ \\
\hline $60-69$ & 231 & \\
Sex, N (\%) & 218 & $(51.4)$ \\
Female & 206 & $(48.6)$ \\
Male & & $(68.0)$ \\
\hline Specialty, $\mathbf{N}(\%)$ & 298 & $(32.0)$ \\
No & 140 & \\
Yes & & \\
\hline
\end{tabular}

Table 2. Percentage of physicians- reported attitudes and barriers to deprescribing.

\begin{tabular}{|c|c|c|c|c|c|c|c|}
\hline Item & $\begin{array}{l}\text { Strongly } \\
\text { disagree } \\
1\end{array}$ & 2 & 3 & 4 & 5 & 6 & $\begin{array}{l}\text { Strongly } \\
\text { agree } \\
7\end{array}$ \\
\hline Q1. From a clinical standpoint, I feel confident with deprescribing in my elderly patients & 0.9 & 2.1 & 5.3 & 22.6 & 30.1 & 26.3 & 12.8 \\
\hline $\begin{array}{l}\text { Q2. When the life expectancy of my elderly patients no longer justifies potential benefits, } \\
\text { I am in favor of deprescribing preventive medications }\end{array}$ & 2.1 & 2.7 & 4.8 & 7.5 & 14.6 & 30.4 & 37.9 \\
\hline $\begin{array}{l}\text { Q3. In elderly patients with poor life expectancy, it would be appropriate to consider } \\
\text { deprescribing therapeutic medications even though they are recommended by guidelines }\end{array}$ & 5.5 & 9.7 & 12.9 & 16.6 & 19.8 & 22.4 & 13.1 \\
\hline $\begin{array}{l}\text { Q4. In elderly patients, lack of robust evidence in favor of continuation or cessation } \\
\text { of preventive medications prevents me from deprescribing }\end{array}$ & 9.2 & 15.0 & 16.8 & 21.0 & 16.6 & 15.4 & 6.0 \\
\hline $\begin{array}{l}\text { Q5. In my elderly patients, I have no hesitation in deprescribing medications initially } \\
\text { prescribed by another physician }\end{array}$ & 7.8 & 9.4 & 10.5 & 17.4 & 16.7 & 23.7 & 14.6 \\
\hline $\begin{array}{l}\text { Q6. I do not have the necessary time to spend with my elderly patients and/or caregivers } \\
\text { to effectively undertake the process of deprescribing medications even though } \\
\text { I consider it important }\end{array}$ & 20.6 & 21.5 & 15.3 & 13.9 & 13.7 & 10.9 & 4.2 \\
\hline $\begin{array}{l}\text { Q7. I have no problem in deprescribing medications even if my elderly patients and/or } \\
\text { caregivers believe continuation is needed }\end{array}$ & 4.8 & 10.3 & 12.6 & 21.1 & 18.3 & 22.7 & 10.3 \\
\hline $\begin{array}{l}\text { Q8. Although in certain situations I may consider appropriate deprescribing medications } \\
\text { in my elderly patients, I do not consider it for fear of adverse drug withdrawal effects }\end{array}$ & 13.1 & 20.5 & 17.5 & 17.5 & 15.6 & 13.1 & 2.8 \\
\hline $\begin{array}{l}\text { Q9. I have no difficulty to motivate my elderly patients and/or caregivers in order to engage } \\
\text { them in the process of deprescribing medications }\end{array}$ & 2.3 & 4.1 & 7.6 & 11.9 & 21.1 & 33.2 & 19.9 \\
\hline
\end{tabular}

For this manuscript, the original survey instrument developed in Italian has been translated into English according to the WHO guidelines (source: http://www.who.int/substance_abuse/research_tools/translation/en/) 
statements regarding preventive medications (second and fourth) one could argue that the very high acceptance of the principle clashes with the perceived lack of information regarding timelines and manner of deprescribing in traditional guidelines. Another explanation could be the fear of adverse effects brought by withdrawal, as well as the fact that the therapy might have been suggested by another doctor.

Admittedly, prescriptions made by different doctors who have no direct contact between themselves for the same patient is regarded in the literature as a risk factor for polypharmacy and is considered an obstacle to deprescribing. ${ }^{20-24}$ Considering statements 1, 2, 6 and 9, doctors appear to agree with the concept of deprescribing (statement 2) and think that they have the necessary understanding (statement 1), the ability to communicate with and motivate their patients (statement 9) and the time to do it (statement 6) but are prevented from applying it because of various external factors. This observation confirms a survey presented to pharmacists, nurses, and doctors by Kouladian which demonstrated the population's tendency to attribute to other professionals rather than granting themselves the responsibility of deprescribing. ${ }^{22}$ This same attitude has been identified as the cause of prescriptive impropriety by Howard Brody who, launching the top five list provocation, called all healthcare professionals to evaluate what can be changed in one's approach and in one's own field, not in someone else's. ${ }^{25,26}$
Almost $45 \%$ of doctors (statement 3 ) hesitate to deprescribe therapeutic medications that are recommended by guidelines, despite the fact that the elderly, those with more than one condition and those who are more likely to be affected by polypharmacy are underrepresented in the case studies that the guidelines are based on. ${ }^{9}$

Similarly to the results of the previous study conducted in the Parma LHA, ${ }^{19}$ in our study almost half of the doctors agree on the fact that the faith in medication that patients and caregivers have represents an obstacle to the implementation of deprescribing. Throughout the study, it is highlighted that patients and caregivers fear adverse effects due to withdrawal of medication and claim that there are more benefits than risks in continuing polypharmacy. ${ }^{25,26}$

Table 3. Percentage of physician Agree (answer 5, 6 or 7 to item).

\begin{tabular}{|c|c|c|}
\hline \multirow[t]{2}{*}{ Item } & \multicolumn{2}{|c|}{ Agree } \\
\hline & $\mathbf{N}$ & $\%$ \\
\hline Q1. From a clinical standpoint, I feel confident with deprescribing in my elderly patients & 303 & 69.2 \\
\hline $\begin{array}{l}\text { Q2. When the life expectancy of my elderly patients no longer justifies potential benefits, I am in favor of deprescribing preventive } \\
\text { medications }\end{array}$ & 363 & 82.9 \\
\hline $\begin{array}{l}\text { Q3. In elderly patients with poor life expectancy, it would be appropriate to consider deprescribing therapeutic medications even though } \\
\text { they are recommended by guidelines }\end{array}$ & 240 & 55.3 \\
\hline $\begin{array}{l}\text { Q4. In elderly patients, lack of robust evidence in favor of continuation or cessation of preventive medications prevents me from } \\
\text { deprescribing }\end{array}$ & 165 & 38.0 \\
\hline Q5. In my elderly patients, I have no hesitation in deprescribing medications initially prescribed by another physician & 241 & 55.0 \\
\hline $\begin{array}{l}\text { Q6. I do not have the necessary time to spend with my elderly patients and/or caregivers to effectively undertake the process } \\
\text { of deprescribing medications even though I consider it important }\end{array}$ & 124 & 28.7 \\
\hline Q7. I have no problem in deprescribing medications even if my elderly patients and/or caregivers believe continuation is needed & 224 & 51.3 \\
\hline $\begin{array}{l}\text { Q8. Although in certain situations I may consider appropriate deprescribing medications in my elderly patients, I do not consider } \\
\text { it for fear of adverse drug withdrawal effects }\end{array}$ & 137 & 31.5 \\
\hline $\begin{array}{l}\text { Q9. I have no difficulty to motivate my elderly patients and/or caregivers in order to engage them in the process of deprescribing } \\
\text { medications }\end{array}$ & 324 & 74.1 \\
\hline
\end{tabular}

Table 4. Correlation between question related to physicians' confidence about deprescribing (Q1) and questions related to physicians' attitudes/barriers (Q2-Q9).

\begin{tabular}{|c|c|c|}
\hline & RHO & $\mathbf{P}$ \\
\hline $\begin{array}{l}\text { Q2. When the life expectancy of my elderly patients no longer justifies potential benefits, I am in favor of deprescribing preventive } \\
\text { medications }\end{array}$ & 0.25 & $<0.01$ \\
\hline $\begin{array}{l}\text { Q3. In elderly patients with poor life expectancy, it would be appropriate to consider deprescribing therapeutic medications even } \\
\text { though they are recommended by guidelines }\end{array}$ & 0.21 & $<0.01$ \\
\hline $\begin{array}{l}\text { Q4. In elderly patients, lack of robust evidence in favor of continuation or cessation of preventive medications prevents me from } \\
\text { deprescribing }\end{array}$ & 0.01 & 0.83 \\
\hline Q5. In my elderly patients, I have no hesitation in deprescribing medications initially prescribed by another physician & 0.33 & $<0.01$ \\
\hline $\begin{array}{l}\text { Q6. I do not have the necessary time to spend with my elderly patients and/or caregivers to effectively undertake the process } \\
\text { of deprescribing medications even though I consider it important }\end{array}$ & -0.02 & 0.71 \\
\hline Q7. I have no problem in deprescribing medications even if my elderly patients and/or caregivers believe continuation is needed & 0.25 & $<0.01$ \\
\hline $\begin{array}{l}\text { Q8. Although in certain situations I may consider appropriate deprescribing medications in my elderly patients, I do not consider it } \\
\text { for fear of adverse drug withdrawal effects }\end{array}$ & -0.08 & 0.09 \\
\hline $\begin{array}{l}\text { Q9. I have no difficulty to motivate my elderly patients and/or caregivers in order to engage them in the process of deprescribing } \\
\text { medications }\end{array}$ & 0.33 & $<0.01$ \\
\hline
\end{tabular}




\section{Implications for research and/or practice}

Research suggests

that recommendations from general practitioners has a positive effect on patients' fears ${ }^{27}$ and that, therefore, they can feel at ease engaging in a conversation with patients regarding deprescribing. This process can be supported by ongoing education regarding how to implement deprescribing in practice, incorporating deprescribing in university curricula and the use of evidence-based, deprescribing instruments. ${ }^{28}$

There are many activities that can be considered to implement deprescribing. We maintain that it is important to spread and increase knowledge, ${ }^{26}$ as we have tried to do with our educational projects addressed to accomplished and prospective general practitioners. The educational project, using a clinical case of a potential 79-year-old woman with the five most common comorbidities was formulated to increase knowledge and the amount of scientific evidence about deprescribing and at the same time recognize and evaluate barriers to enacting it in a simulated practice-based setting. There are validated criteria identifying potentially inappropriate medications, such as the Beers criteria ${ }^{29}$ and STOPP/START criteria, ${ }^{30}$ that may facilitate the deprescribing process, as well as practical instruments such as the Canadian website Deprescribing.org (https:// deprescribing.org/). ${ }^{31}$ Educational seminars with small groups can help doctors face the conversation with patients in order to reassure them and conversations with colleagues in order to obtain efficient coordination of medication prescription.

\section{Conclusions}

In conclusion, the present study confirms that general practitioners operating in Turin sense the importance of deprescribing and feel prepared to face it managing communication with patients and caregivers, but find barriers when enacting the practice in a real-life context. Amongst the mentioned barriers, time management seems to be a minor problem, while other barriers (prescription by a colleague, disagree with the opinion of the patient or the care giver, absence of strong evidence supporting deprescribing, fear of possible effects due on suspension) are in analogy with those found in the literature. We think that a comparison with specialists is necessary in order to share a way to tackle polypharmacy. The involvement of health care organizations is also crucial. Our project therefore now involves disseminating our data to primary care colleagues and specialists and continuing training events for GPs.

\section{References}

1. Wise J. Polypharmacy: a necessary evil. BMJ 2013;347:f7033.

2. Reeve E, Thompson W, Farrell B. Deprescribing: a narrative review of the evidence and practical recommendations for recognizing opportunities and taking action. Eur J Intern Med 2017;38:3-11.

3. Woodward MC. Deprescribing: achieving better health outcomes for older people through reducing medications. J Pharm Res 2003;33:323-8.

4. Marengoni A, Onder G. Guidelines, polypharmacy, and drug-drug interactions in patients with multimorbidity. A cascade of failure BMJ 2015;350:h1059.

5. Woolf SH, Grol R, Hutchinson A, et al. Potential benefits, limitations, and harms of clinical guidelines. BMJ 1999;318: 527-30

6. Hibble A, Kanka D, Pencheon D, Pooles F. Guidelines in general practice: the new Tower of Babel? BMJ 1998;317: 862-3.

7. Pizzini A. Prescription of drugs in multimorbidity: when is it too much? J AMD 2018;21:14-20

8. Page A, Clifford R, Potter K, EthertonBeer C. A concept analysis of deprescribing medications in older people. J Pharm Pract Res 2018;48:132-48.

9. Kuehlein T, Sghedoni D, Visentin G, Gérvas J, Jamoulle M. Quaternary prevention: a task of the general practitioner. Primary Care 2010, 18. available at: https://orbi.uliege.be/ browse?type $=$ journal $\&$ value $=$ Primary + Care (accessed March 7, 2020).

10. Widmer D, Herzig L, Jamoulle M. Prévention quaternaire: agir est-il toujours justifié en médecine de famille? [Quaternary prevention: is acting always justified in family medicine?]. Rev Med Suisse 2014; 10:1052-6.

11. Jamoulle M. Quaternary prevention, an answer of family doctors to over medicalization. Int $\mathrm{J}$ Health Policy Manag 2015;4:61-4.

12. Kua CH, Mak VSL, Lee SVH. Health outcomes of deprescribing interventions among older residents in nursing homes: a systematic rewiev and meta-analysis. Jamda 2019;20:362-72.

13. Garfinkel G, Mangin D. Feasibility Study of a Systematic Approach for Discon- tinuation of Multiple Medications in Older Adults: Addressing Polypharmacy. Arch Intern Med 2010;170:1648-54.

14. Scott IA, Hilmer SN, Reeve E, et al. Reducing inappropriate polypharmacy: the process of deprescribing. JAMA Intern Med 2015;175:827-34.

15. Reeve E, To J, Hendrix I, et al. Patient barriers to and enablers of deprescribing: a systematic review. Drugs Aging 2013;30:793-807.

16. Anderson K, Stowasser D, Freeman C, Scott I. Prescriber barriers and enablers to minimising potentially inappropriate medications in adults: a systematic review and thematic synthesis. BMJ Open 2014;4:e006544-2014-006544.

17. Zechmann S, Trueb C, Valeri F, et al. Barriers and enablers for deprescribing among older, multimorbid patients with polypharmacy: an explorative study from Switzerland. BMC Fam Pract 2019;20:64.

18. Mantelli S, Jungo KT, Rozsnyai Z, et al. How general practitioners would deprescribe in frail oldest-old with polypharmacy - the LESS study. BMC Fam Pract 2018;19:169.

19. Djatche L, Lee S, Singer D, et al. How confident are physicians in deprescribing for the elderly and what barriers prevent deprescribing? J Clin Pharm Ther 2018; 43:550-5.

20. Bokhof B, Junius-Walker U. Reducing polypharmacy from the perspectives of general practitioners and older patients: a synthesis of qualitative studies. Drugs Aging 2016;33:249-66.

21. Clyne B, Cooper JA, Hughes CM, et al. OPTI-SCRIPT study team. 'Potentially inappropriate or specifically appropriate?' qualitative evaluation of general practitioners views on prescribing, polypharmacy and potentially inappropriate prescribing in older people. BMC Fam Pract 2016; 17:109.

22. Ailabouni NJ, Nishtala PS, Mangin D, Tordoff JM. Challenges and enablers of deprescribing: a general practitioner perspective. PLoS One 2016;11: e0151066.

23. Kouladjian L, Gnjidic D, Reeve E, et al. Health care practitioners' perspectives on deprescribing anticholinergic and sedative medications in older adults. Ann Pharmacother 2016;50:625-36.

24. Scott IA, Gray LC, Martin JH, Mitchell CA. Minimizing inappropriate medications in older populations: a 10 step conceptual framework. Am J Med 2012;125:529-37.e4

25. Reeve E, Low LF, Hilmer SN. Beliefs and attitudes of older adults and carers 
about deprescribing of medications: a qualitative focus group study. Br J Gen Pract 2016;66:e552-60.

26. Luymes $\mathrm{CH}$, van der Kleij RM, Poortvliet RK, et al. Deprescribing potentially inappropriate preventive cardiovascular medication: barriers and enablers for patients and general practitioners. Ann Pharmacother 2016;50:446-54.

27. Brody H. Medicine's Ethical Responsibi- lity for Health Care Reform - The Top Five List. N Engl J Med 2010;362:283-5.

28. Djatche L, Singer D, Heyer A, et al. How can we effectively engage physicians in the deprescribing process? Am J Med Qual 2017;32:581-2.

29. Beers MH. Explicit criteria for determining potentially inappropriate medication use by the elderly. An update. Arch Intern Med 1997;157:1531-6.
30. O'Mahony D, Gallagher P, Ryan C, et al. STOPP \& START criteria: A new approach to detecting potentially inappropriate prescribing in old age. Eur Ger Med 2010;1:45-51.

31. Boyd CM, Darer J, Boult C, et al. Clinical practice guidelines and quality of care for older patients with multiple comorbid diseases. JAMA 2005;294: 716-24. 